Open Access

\title{
Impact of add-on laboratory testing at an academic medical center: a five year retro- spective study
}

Louis S. Nelson ${ }^{1}$, Scott R. Davis ${ }^{1}$, Robert M. Humble ${ }^{1}$, Jeff Kulhavy ${ }^{1}$, Dean R. Aman² and Matthew D. Krasowski ${ }^{*}$

\begin{abstract}
Background: Clinical laboratories frequently receive orders to perform additional tests on existing specimens ('add-ons'). Previous studies have examined add-on ordering patterns over short periods of time. The objective of this study was to analyze add-on ordering patterns over an extended time period. We also analyzed the impact of a robotic specimen archival/retrieval system on add-on testing procedure and manual effort.

Methods: In this retrospective study at an academic medical center, electronic health records from were searched to obtain all add-on orders that were placed in the time period of May 2, 2009 to December 31, 2014.

Results: During the time period of retrospective study, 880,359 add-on tests were ordered on 96,244 different patients. Add-on testing comprised $3.3 \%$ of total test volumes. There were 443,411 unique ordering instances, leading to an average of 1.99 add-on tests per instance. Some patients had multiple episodes of add-on test orders at different points in time, leading to an average of 9.15 add-on tests per patient. The majority of add-on orders were for chemistry tests (78.8\% of total add-ons) with the next most frequent being hematology and coagulation tests (11.2\% of total add-ons). Inpatient orders accounted for $66.8 \%$ of total add-on orders, while the emergency department and outpatient clinics had $14.8 \%$ and $18.4 \%$ of total add-on orders, respectively. The majority of add-ons were placed within 8 hours ( $87.3 \%$ ) and nearly all by 24 hours (96.8\%). Nearly $100 \%$ of add-on orders within the emergency department were placed within 8 hours. The introduction of a robotic specimen archival/retrieval unit saved an average of 2.75 minutes of laboratory staff manual time per unique add-on order. This translates to 24.1 hours/day less manual effort in dealing with add-on orders.
\end{abstract}

Conclusion: Our study reflects the previous literature in showing that add-on orders significantly impact the workload of the clinical laboratory. The majority of add-on orders are clinical chemistry tests, and most add-on orders occur within 24 hours of original specimen collection. Robotic specimen archival/retrieval units can reduce manual effort in the clinical laboratory associated with add-on orders.

Keywords: Clinical chemistry tests, Clinical laboratory information services, Clinical laboratory services, Hematology, Laboratory automation, Robotics

\footnotetext{
* Correspondence: mkrasows@healthcare.uiowa.edu

${ }^{1}$ Department of Pathology, University of lowa Hospitals and Clinics, lowa

City, IA 52242, USA

Full list of author information is available at the end of the article
}

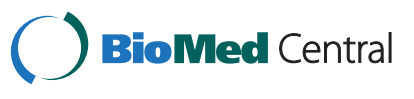

(c) 2015 Nelson et al.; licensee BioMed Central. This is an Open Access article distributed under the terms of the Creative Commons Attribution License (http://creativecommons.org/licenses/by/4.0), which permits unrestricted use, distribution, and reproduction in any medium, provided the original work is properly credited. The Creative Commons Public Domain Dedication waiver (http://creativecommons.org/publicdomain/zero/1.0/) applies to the data made available in this article, unless otherwise stated. 


\section{Background}

Clinical laboratories frequently receive orders to perform additional tests on existing specimens ('add-ons'). Melanson et al. in 2004 was the first published report analyzing the operational impact of add-on testing, demonstrating patterns of misutilization (e.g., failure to follow laboratory testing algorithms in institutional chest pain protocols) in a significant fraction of add-on orders [1]. A follow-up study in 2006 compared add-on testing between two academic hospitals, showing similarities in add-on ordering patterns and proposing strategies to improve the process [2]. There have been several other studies on add-on test ordering, each analyzing less than one month of add-on orders [3-5].

In this study at an academic medical center, we retrospectively analyzed add-on testing data over a five and a half year period (May 2009- Dec 2014). This allowed for the analysis of add-on ordering trends over a much longer period of time than in previous studies. Also, during this time period, the core clinical laboratory of the institution introduced a robotic archival specimen retrieval system that changed the add-on testing procedure. We analyzed the impact of this unit on add-on testing procedure and manual workload.

\section{Methods}

\section{Institutional setting}

The study was approved by the University of Iowa Institutional Review Board as a retrospective study covering the time period from May 2, 2009- December 31, 2014. In this large retrospective study, there was waiver of informed consent and authorization approved by the Institutional Review Board for all subjects. The institution in this study is the University of Iowa Hospitals and Clinics (UIHC), a 730 bed academic medical center that includes an emergency department (ED) with level one trauma capability, adult and pediatric inpatient floors, and multiple intensive care units (ICUs; neonatal, pediatric, medical, cardiovascular, and neurologic/surgical). Outpatient services are located at the main medical campus in Iowa City, IA, as well as at a multispecialty outpatient facility located three miles away. Smaller primary care clinics are located throughout the local region. A core clinical laboratory within the Department of Pathology provides clinical chemistry and hematopathology testing. Two critical care laboratories (one located near the main operating rooms and another embedded within the neonatal ICU) perform blood gas and activated clotting time testing. There are also separate clinical laboratories for anatomic pathology, blood center, and microbiology/molecular pathology located within the main medical campus.

\section{Hospital and laboratory informatics}

The electronic health record (EHR) for UIHC was Epic (Epic Systems, Inc, Madison, WI). Computerized provider order entry (CPOE) is available in Epic to licensed independent providers. Add-on orders can be placed within the
EHR by CPOE or by calling the laboratory. Throughout the period of retrospective study, providers were directed, when feasible, to place orders within the EHR and limit the number of verbal orders requiring laboratory-initiated testing orders. In general, chemistry and hematology tests are all orderable individually. However, there are some panels built in Epic: basic metabolic panel with total calcium (BMP; sodium, chloride, carbon dioxide, potassium, blood urea nitrogen, creatinine, glucose, total calcium), electrolyte panel (sodium, chloride, carbon dioxide, potassium), complete blood count (CBC; white blood cell count, hemoglobin, hematocrit, red blood cell count, platelet count), and lipid panel (total cholesterol, high-density lipoprotein, triglycerides, calculated low-density lipoprotein). For the purposes of analysis in this manuscript, panels were broken apart into individual tests except where described otherwise. Categories of testing were also defined (Table 1) to provide better comparison to other published studies on add-on testing $[1,2,5]$.

The laboratory information system (LIS) for all UIHC pathology laboratories until August 2, 2014 was Cerner (Kansas City, MO, USA) "Classic”, currently version 015. On August 2, 2014, the clinical pathology laboratories switched to Epic Beaker as the LIS, retaining Cerner as the LIS for anatomic pathology, blood center, and some parts of hematopathology and molecular pathology. The switch to Epic Beaker allowed for accurate capture of the timing of add-on orders relative to when the original specimen was received in the laboratory. During this nearly 5 month period (August 2 to December 31, 2014), there were 56,389 add-on orders with complete time data.

\section{Laboratory instrumentation and add-on testing procedures}

The instrumentation and informatics within the core laboratory of UIHC has been described in detail in previous reports $[6,7]$. Throughout the time period of retrospective analysis, the main chemistry instrumentation in the core laboratory was from Roche Diagnostics (Indianapolis, IN, USA). Front-end automation was provided by a Modular Pre-Analytic (MPA)-7 unit. In February 2014, the core laboratory went live with a Roche Diagnostics P701 automated archival/retrieval system. This system changed the add-on process (Fig. 1). Originally, specimens were placed into archival racks by the instrument flexible sample sorters. The most recent racks were kept near the instruments. The racks were then archived manually to a set of refrigerators for storage for 3 to 5 days (dependent on available refrigerator space and number of specimens). The P701 automated the sample storage and retrieval process (Fig. 1b), eliminating manual steps.

The manual effort involved from the moment the add-on is printed into the laboratory to the time the sample is 
Table 1 Abbreviations for assay categories

\begin{tabular}{|c|c|c|}
\hline Abbreviation & Full Name & Test(s) Included \\
\hline $\mathrm{A} 1 \mathrm{C}$ & Hemoglobin A1C & Hemoglobin A1C \\
\hline ANEMIA & Anemia Testing & Iron, total iron-binding capacity, ferritin, folate, vitamin $B_{12}$ \\
\hline BILD & Bilirubin, Direct & Direct (conjugated) bilirubin \\
\hline BMP & Basic Metabolic Panel & $\begin{array}{l}\text { Sodium, potassium, chloride, carbon dioxide, blood urea nitrogen, } \\
\text { creatinine, glucose, and calcium }\end{array}$ \\
\hline CARDIAC & Cardiac Markers & Creatine kinase- $\mathrm{MB}$, troponin $\mathrm{T}$, $\mathrm{N}$-terminal B-type natriuretic peptide \\
\hline $\mathrm{CBC}$ & Complete Blood Count & $\begin{array}{l}\text { White blood cell count, red blood cell count, hemoglobin, hematocrit, } \\
\text { platelet count }\end{array}$ \\
\hline CRP & C-Reactive Protein & C-Reactive Protein \\
\hline DIFF & Differential & White blood cell differential \\
\hline ENDO & Endocrinology Testing & $\begin{array}{l}\text { Thyroid-stimulating hormone, thyroxine }\left(T_{4}\right) \text { - total and free, triiodothyronine } \\
\left(T_{3}\right) \text { - total and free, cortisol, testosterone, and } 25 \text {-hydroxyvitamin } D\end{array}$ \\
\hline ESR & Erythrocyte Sedimentation Rate & Erythrocyte sedimentation rate \\
\hline GASES & Blood Gas Analyzer Laboratory Studies ${ }^{a}$ & $\begin{array}{l}\text { Lactic acid, potassium, glucose, hemoglobin, hematocrit, sodium, chloride, ionized } \\
\text { calcium, } \mathrm{pO}_{2}, \mathrm{pCO}_{2} \text {, oxygen saturation, methemoglobin, carboxyhemoglobin }\end{array}$ \\
\hline HAPT & Haptoglobin & Haptoglobin \\
\hline HEPC & Hepatitis C Antibody & Hepatitis C antibody \\
\hline HBSG & Hepatitis B Surface Antigen & Hepatitis B surface antigen \\
\hline LFP & Liver Function Panel & $\begin{array}{l}\text { Albumin, alkaline phosphatase, total bilirubin, total protein, alanine aminotransferase, } \\
\text { aspartate aminotransferase, and } \gamma \text {-glutamyltranspeptidase }\end{array}$ \\
\hline $\mathrm{LDH}$ & Lactate Dehydrogenase & Lactate dehydrogenase \\
\hline REFERENCE & Reference Laboratory Testing & All testing referred to external reference laboratory \\
\hline OSMO & Osmolality & Serum and urine osmolality \\
\hline PO4MG & Phosphorus and Magnesium & Phosphorus, magnesium \\
\hline PREALB & Prealbumin & Prealbumin \\
\hline PT/INR & Prothrombin Time/INR & Prothrombin time/International normalized ratio \\
\hline PTT & Partial Thromboplastin Time & Partial thromboplastin time \\
\hline RETIC & Reticulocytes & Reticulocytes \\
\hline TAP & Toxic Alcohol Panel & Sodium, glucose, blood urea nitrogen, osmolality, ethanol \\
\hline URIC & Uric Acid & Uric acid \\
\hline
\end{tabular}

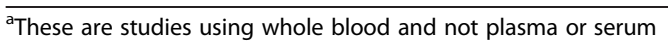

loaded on the proper analyzer averaged approximately 3.5 minutes prior to introduction of the P701 (composed in large part in retrieving specimens from racks near instruments or in the refrigerators), and 45 seconds once the P701 was implemented. In the prior system of manual archiving and retrieval, instances where samples were archived improperly could significantly delay the process.

\section{Results}

\section{Timing trends in add-on testing}

In the time period of retrospective study (May 2, 2009 to December 31, 2014), there were a total of 880,359 add-on orders at UIHC. This comprised $3.3 \%$ of the total laboratory test volume performed within the clinical laboratories. The total number of add-on orders increased every year from 2009 to 2013 and then decreased slightly in 2014 (Fig. 2a, b). Fig. 2c shows the variation of add-on orders by month. The inpatient population had the majority of add-on orders (66.8\%), while the ED and outpatient clinics accounted for $14.8 \%$ and $18.4 \%$ of add-on orders, respectively.

Capture of the exact timing of add-on orders relative to initial specimen collect was only possible with the new LIS (August 2, 2014 - December 31, 2014; complete data available on 56,389 add-on orders). Fig. 3a plots the timing of add-on orders, showing the percentage within periods of time. The majority of add-ons were placed within 8 hours $(87.3 \%)$ and nearly all by 24 hours $(96.8 \%)$. The timing of ordering varied by patient location. Add-on orders placed for patients in the ED were generally closer to original specimen collect time as compared to inpatient units and outpatient clinics. Nearly $100 \%$ of add-on orders within 


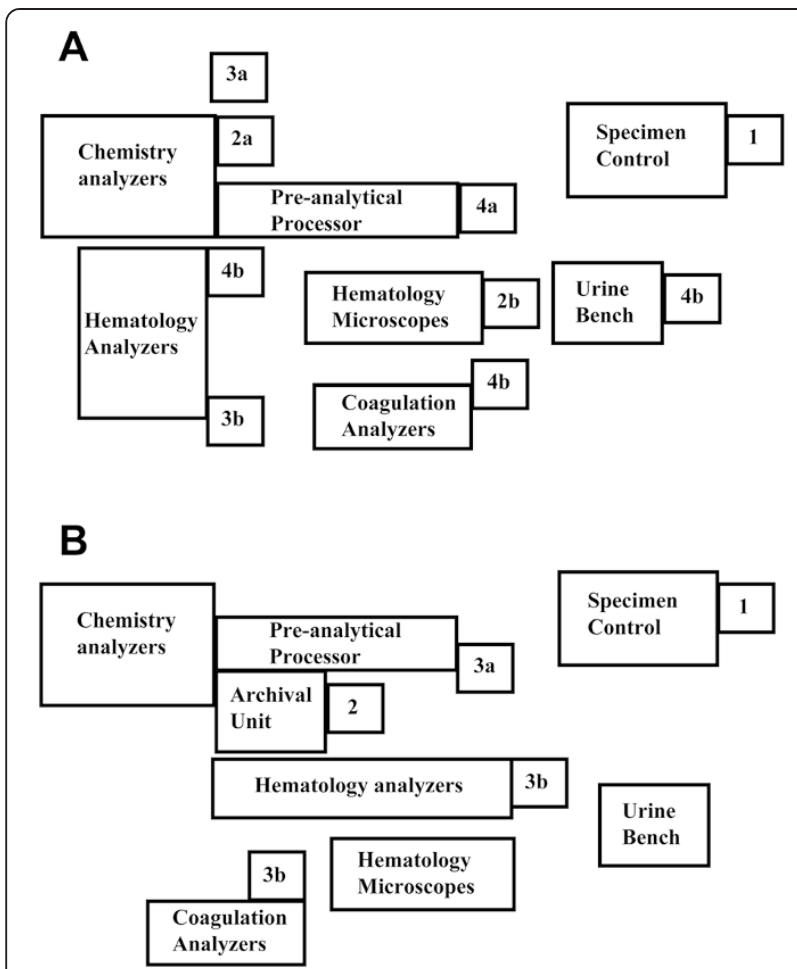

Fig. 1 Add-on Testing Procedure. a Layout of the core laboratory prior to the automated specimen archival/retrieval unit. Add-on orders generate a print-out in the core laboratory with the testing information and patient demographics (1). A laboratory assistant reviews the print-out. If an add-on can be performed, the assistant prints an additional label with test information to a designated area of the laboratory (e.g., chemistry, 2a, or hematology, 2b) depending on the add-on order. A technologist enters in accession number into the computer program that tracks the archival rack and position for the specimen. Then technologist retrieves the specimen from the archival rack (3a or $3 b$ ) or from the refrigerator. The technologist loads specimen on proper analyzer (4a, 4b). $\mathbf{b}$ Layout of the core laboratory after the automated specimen archival/retrieval unit. Similar to above, add-on orders generate a print-out in the core laboratory that is reviewed by laboratory assistant (1). If an add-on can be performed, the assistant then uses a computer program to request the specimen be retrieved from the archiver (1). The archiver then locates the specimen and dispenses it (2). The assistant retrieves the specimen from archiver and loads specimen on to proper analyzer (3a or $3 b)$

the ED were placed within 8 hours. Timing of add-on orders for inpatient units and outpatient clinics were similar, except that there was a longer tail of add-on orders placed 24 hours or more later than original specimen collect in the outpatient population (Fig. 3a).

The peak times for add-on order placement were between 08:00 - 12:00, with $47.8 \%$ of add-ons ordered between 07:00 and 13:00 (Fig. 3b). Add-on orders were more frequent during weekdays, with Saturdays and Sundays only accounting for slightly more than $20 \%$ of total add-on volume (Fig. 3c).

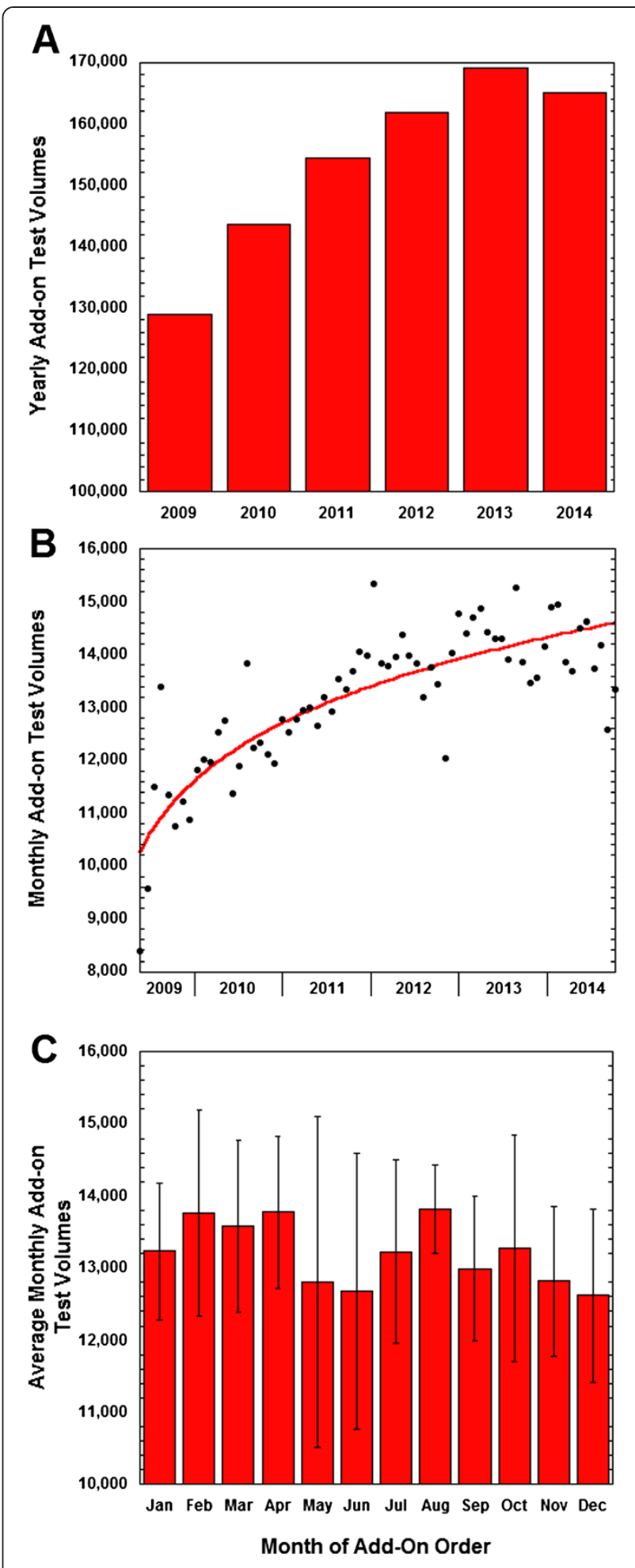

Fig. 2 Add-on Order Volumes. a Yearly add-on testing volumes from 2009 - 2014. The data in 2009 is normalized to an entire year based on order volume from May 2, 2009 to end of that calendar year. $\mathbf{b}$ Monthly add-on testing volumes spanning 2009 - 2014. c Add-on test volumes per month 

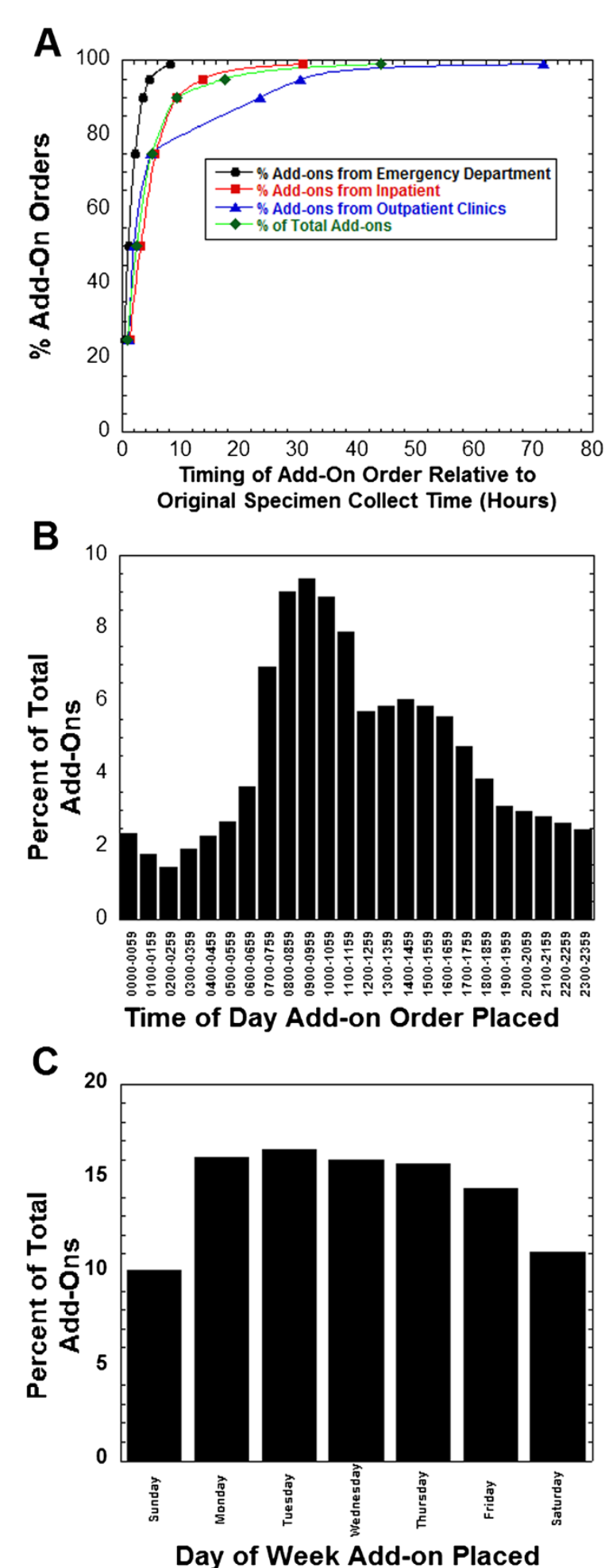

Fig. 3 Timing of Add-on Orders. a Timing of add-on orders relative to original specimen collect time. The data is broken down into orders originating from emergency department (ED), inpatient units (including intensive care units), outpatient clinics, and all data. b Time of day add-on order was placed broken into one hour intervals. c Day of week add-on order was placed
Distribution of add-on testing by category of testing

Figure 4 shows a breakdown of add-on testing by category of testing. The majority of the add-on tests were chemistry tests $(78.8 \%$ of total add-on orders comprising $3.3 \%$ of overall chemistry test volumes), with the most frequent being within the following categories (all percentages are of the total overall add-on orders): LFP (25.6\%), BMP (11.7\%), MGPO4 (7.0\%), Cardiac (4.6\%), and Anemia (3.2\%). Hematology and coagulation tests were the next most frequent areas of add-on testing. The most frequent hematology and coagulation add-ons were in the following categories (all percentages are of the total overall add-on orders): CBC (2.70 \%), Diff (2.19\%), PTT (1.19\%), PT/ INR (1.71\%), and ESR (0.85\%). Within the critical care laboratories, the most frequent add-on orders were the following tests performed on blood gas analyzers using whole blood specimens: lactic acid $(0.97 \%)$, potassium (0.78 \%), glucose (0.62 \%), hemoglobin/hematocrit (0.49\%), and sodium (0.49\%). Urinalysis-related tests accounted for less than $1 \%$ of total add-ons. For the critical care laboratory tests, in most cases the parameters had already been determined as part of a cartridge of testing on the blood gas analyzers; results were suppressed from reporting to the LIS if not ordered by the provider. Thus, the add-on order for these tests required only that staff perform the computer steps necessary to release the previously unordered test from the instrument to the LIS.

Table 2 summarizes the most frequent add-on orders broken down into categories of testing. LFP, BMP, MGPO4, ENDO, and CARDIAC ranked in the top ten

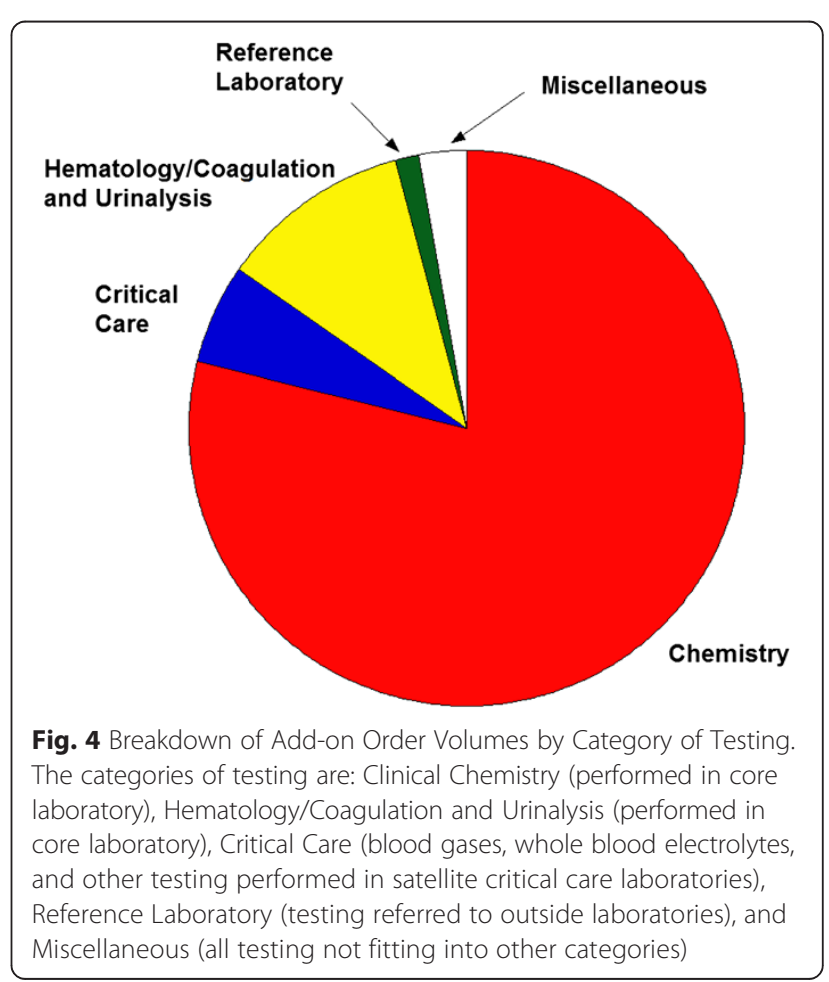


most frequent add-on orders overall and also individually for inpatient units, ED, and outpatient clinics. Not surprisingly, GASES were an infrequent add-on order in the outpatient clinics but were the fourth most common overall add-on order. A1C was more frequently addedon in the outpatient clinics than from the ED or inpatient units. Table 3 lists the most frequent individual tests added-on. The top five most frequent individual add-on tests were magnesium (4.0\%), albumin (3.8\%), alanine aminotransferase (3.8\%), aspartate aminotransferase (3.8\%), and total bilirubin (3.7 \%). Table 3 also lists the percentage of times each individual test was ordered as an add-on. For six of the tests (amylase, creatine kinase, hemoglobin $\mathrm{A} 1 \mathrm{C}$, lactate dehydrogenase, lipase, troponin $\mathrm{T}$ ), over $10 \%$ of orders for that particular test were placed as add-ons.

Reference laboratory testing comprised only $1.4 \%$ of total add-ons, with no single test accounting for more than $0.05 \%$ of total add-ons (Table 4). There were 618 different reference laboratory tests available in the EHR test menu that were ordered at least once as an add-on in the time period of retrospective study. This included 193 orders of "Miscellaneous Test", an order option in the EHR for reference laboratory testing not built in the EHR test menu. Our analysis does not capture attempts to order reference laboratory testing that could not be completed due to lack of suitable existing specimen.

\section{Workload impact of add-on testing}

Table 5 summarizes the workload of add-on testing over the period of retrospective study. Of the 880,359 add-on orders, there were 443,411 unique ordering instances, leading to an average of 1.99 add-on tests per instance. Some patients had multiple episodes of add-on test orders at different points in time (e.g., during different days of a multi-day inpatient encounter), leading to an average of 9.15 add-on tests per patient. The introduction of the robotic specimen archival/retrieval unit saved an average of 2.75 mins of laboratory staff manual time per unique ordering instance (Table 5). This translates to $24.1 \mathrm{hr} /$ day less manual effort in dealing with add-on orders.

\section{Discussion}

Add-on testing can occupy a significant amount of clinical laboratory resources $[1,3-5]$. The main challenges are storage of specimens and the labor involved in retrieving specimens for further testing $[1,5]$. Add-on testing can theoretically serve a useful purpose in allowing for thoughtful ordering of additional testing based on initial laboratory test results or other clinical data. On the other

Table 2 Most frequently ordered add-on test categories by ordering location

\begin{tabular}{|c|c|c|c|c|c|c|c|c|}
\hline \multirow[b]{2}{*}{ Test Category } & \multicolumn{2}{|c|}{ Inpatient Unit } & \multicolumn{2}{|c|}{ Emergency Department } & \multicolumn{2}{|c|}{ Outpatient Clinics } & \multicolumn{2}{|c|}{ All Locations } \\
\hline & $\%$ & Rank & $\%$ & Rank & $\%$ & Rank & $\%$ & $\overline{\text { Rank }}$ \\
\hline LFP & $28.79 \%$ & 1 & $29.86 \%$ & 1 & $16.91 \%$ & 1 & $26.76 \%$ & 1 \\
\hline BMP & $12.00 \%$ & 2 & $10.14 \%$ & 2 & $15.12 \%$ & 2 & $12.30 \%$ & 2 \\
\hline MGPO4 & $7.41 \%$ & 4 & $4.02 \%$ & 4 & $2.87 \%$ & 5 & $6.08 \%$ & 3 \\
\hline GASES & $8.41 \%$ & 3 & $2.51 \%$ & 9 & $0.10 \%$ & 19 & $6.01 \%$ & 4 \\
\hline ENDO & $3.30 \%$ & 6 & $3.09 \%$ & 6 & $8.81 \%$ & 3 & $4.28 \%$ & 5 \\
\hline CARDIAC & $4.19 \%$ & 5 & $7.25 \%$ & 3 & $2.09 \%$ & 7 & $4.25 \%$ & 6 \\
\hline ANEMIA & $2.66 \%$ & 7 & $0.78 \%$ & 18 & $6.67 \%$ & 4 & $3.12 \%$ & 7 \\
\hline DIFF & $2.40 \%$ & 8 & $1.73 \%$ & 15 & $1.78 \%$ & 9 & $2.19 \%$ & 8 \\
\hline $\mathrm{CBC}$ & $2.24 \%$ & 9 & $1.83 \%$ & 14 & $2.00 \%$ & 8 & $2.14 \%$ & 9 \\
\hline PT/INR & $1.43 \%$ & 12 & $3.57 \%$ & 5 & $0.88 \%$ & 13 & $1.65 \%$ & 10 \\
\hline LIPASE & $1.49 \%$ & 11 & $3.01 \%$ & 8 & $0.77 \%$ & 15 & $1.58 \%$ & 11 \\
\hline $\mathrm{A} 1 \mathrm{C}$ & $1.33 \%$ & 14 & $0.95 \%$ & 17 & $2.52 \%$ & 6 & $1.49 \%$ & 12 \\
\hline CRP & $1.29 \%$ & 15 & $2.22 \%$ & 10 & $1.39 \%$ & 11 & $1.45 \%$ & 13 \\
\hline $\mathrm{LDH}$ & $1.50 \%$ & 10 & $0.64 \%$ & 19 & $1.43 \%$ & 10 & $1.36 \%$ & 14 \\
\hline AMYLASE & $1.37 \%$ & 13 & $2.02 \%$ & 12 & $0.68 \%$ & 16 & $1.34 \%$ & 15 \\
\hline PTT & $0.97 \%$ & 16 & $3.03 \%$ & 7 & $0.19 \%$ & 17 & $1.13 \%$ & 16 \\
\hline ESR & $0.61 \%$ & 18 & $2.00 \%$ & 13 & $0.78 \%$ & 13 & $0.85 \%$ & 17 \\
\hline BILD & $0.94 \%$ & 17 & $0.59 \%$ & 20 & $0.92 \%$ & 12 & $0.85 \%$ & 18 \\
\hline D-DIMER & $0.24 \%$ & 19 & $2.07 \%$ & 11 & $0.11 \%$ & 18 & $0.48 \%$ & 19 \\
\hline TAP & $0.16 \%$ & 20 & $1.61 \%$ & 16 & $0.03 \%$ & 20 & $0.35 \%$ & 20 \\
\hline Total & $82.7 \%$ & & $82.9 \%$ & & $66.1 \%$ & & $79.7 \%$ & \\
\hline
\end{tabular}


Table 3 Most frequently ordered individual add-on tests

\begin{tabular}{|c|c|c|}
\hline Test & $\%$ of Total Add-on Orders & $\%$ of Times Test Ordered as Add-on (vs. Routine) \\
\hline Magnesium & 4.0 & 5.9 \\
\hline Albumin & 3.8 & 6.5 \\
\hline Alanine aminotransferase & 3.8 & 5.4 \\
\hline Aspartate aminotransferase & 3.8 & 5.5 \\
\hline Bilirubin, total & 3.7 & 6.4 \\
\hline Alkaline phosphatase & 3.4 & 5.8 \\
\hline Gamma-glutamyltransferase & 3.2 & 4.7 \\
\hline Total protein & 3.1 & 6.3 \\
\hline Phosphorus & 3.0 & 6.9 \\
\hline Troponin T & 2.2 & 14.5 \\
\hline Complete blood count & 2.1 & 1.5 \\
\hline Automated white blood cell differential & 2.1 & 2.3 \\
\hline Creatinine & 1.7 & 1.1 \\
\hline Potassium & 1.6 & 1.1 \\
\hline Thyroid stimulating hormone with reflex to free thyroxine & 1.6 & 9.6 \\
\hline Lipase & 1.6 & 15.9 \\
\hline Hemoglobin A1C & 1.5 & 10.2 \\
\hline Prothrombin time/International normalized ratio & 1.5 & 1.8 \\
\hline Basic metabolic panel with calcium & 1.5 & 1.1 \\
\hline C-Reactive protein & 1.4 & 8.1 \\
\hline Lactic acid dehydrogenase & 1.4 & 10.7 \\
\hline Creatine kinase, total & 1.3 & 22.7 \\
\hline Amylase & 1.3 & 15.3 \\
\hline Blood urea nitrogen & 1.3 & 0.9 \\
\hline Calcium, total & 1.2 & 1.3 \\
\hline
\end{tabular}

Table 4 Most frequently ordered reference laboratory add-on tests

\begin{tabular}{|c|c|}
\hline Test & $\%$ of Total Add-on Orders \\
\hline Helicobacter pylori antibody, IgG & $0.050 \%$ \\
\hline Mitochondrial M2 antibodies & $0.049 \%$ \\
\hline Hepatitis C virus quantitative PCR & $0.033 \%$ \\
\hline lgG subclasses & $0.022 \%$ \\
\hline Miscellaneous test ${ }^{\mathrm{a}}$ & $0.019 \%$ \\
\hline Vitamin D, 1,25-dihydroxy & $0.018 \%$ \\
\hline Aldosterone, serum & $0.017 \%$ \\
\hline Alpha-1-antitrypsin, phenotyping & $0.013 \%$ \\
\hline Lyme disease antibodies, IgG and IgM & $0.013 \%$ \\
\hline Histoplasma antigen, urine & $0.013 \%$ \\
\hline
\end{tabular}

${ }^{a}$ Covers any test ordered that is not on list of laboratory tests built in electronic medical record
Table 5 Summary of add-on tests

\begin{tabular}{ll}
\hline Total number of add-ons & 880,359 \\
\hline Unique ordering instances $^{1}$ & 443,411 \\
Number of patients & 96,244 \\
Average number of add-on tests per unique ordering instance $^{\text {Average number of add-on tests per patient }}{ }^{2}$ & 1.99 \\
Estimated hours per day of manual effort for add-on testing $^{\text {prior to introduction of robotic archival storage/retrieval unit }}$ & $\mathbf{3 0 . 1 5}$ \\
$\begin{array}{l}\text { Estimated hours per day of manual effort for add-on testing } \\
\text { with use of robotic archival storage/retrieval unit }\end{array}$ & 6.6
\end{tabular}

${ }^{1}$ These are unique add-on orders at a specific time ${ }^{2}$ This includes all add-on tests per each patient, which may span multiple unique ordering instances 
hand, add-on testing can also be a result of disorganized ordering practices (e.g., neglecting to order essential laboratory testing upfront) or even misutilization of testing, as has been shown in previous studies [1,2]. Our analysis does not capture attempts at add-on testing that could not be completed due to lack of existing specimens or add-on testing that was duplicate to previously ordered testing (e.g., hemoglobin/hematocrit placed as add-on when $\mathrm{CBC}$ already performed). We have analyzed duplicate testing as an example of misutilization in a previous study [6].

The results of this study are similar to previous studies in showing that the majority of add-ons are clinical chemistry tests $[2,4,5]$. As with previous studies, LFP, BMP, MGPO4, and CARDIAC were in the top tier of ordered add-on tests. Hematology and coagulation testing only accounted for $11.2 \%$ of total add-ons; however, add-ons comprised $5.6 \%$ of the overall hematology/coagulation test volumes. Four common tests (CBC, DIFF, PT/INR, and PT) accounted for over two-thirds of the hematology and coagulation add-on tests.

In the present study, the $5.86 \%$ percent of add-ons for the critical care laboratories posed the least manual effort because in most cases the parameters had already been determined but were suppressed from reporting to the LIS if not ordered by the provider. Therefore, most critical care laboratory add-ons only needed to be sent from instrument to LIS rather than having to locate the sample and analyze it again (assuming specimen would even be viable at that point). Reference laboratory addons were only a small fraction of total add-ons but spanned a wide variety of tests. No single reference laboratory test exceeded $0.05 \%$ of total add-ons. These results are comparable to previous studies [2,5]. Add-on requests for reference laboratory tests not built in the LIS or EHR may entail extra work in first determining the specimen requirements for the requested test and then checking if pre-existing specimens can be used.

Similar to previous studies, our study shows a high fraction of add-ons ordered within eight hours of original specimen collect time $[1,2,5]$. The ED tended to order add-ons more quickly compared to inpatient units and outpatient clinics. Only a small fraction of add-on orders were placed more than 24 hours after original specimen collection. Less than $1 \%$ of total add-ons occurred more than 48 hours after specimen collection. Outpatient clinics accounted for the majority of add-on orders submitted more than 24 hours after original specimen collection, a finding similar to a previous study [5].

Depending on workload, add-on orders can entail substantial manual effort from clinical laboratory staff $[1,2,4,5]$. In our study, add-ons comprised $3.3 \%$ of overall test volume, a figure that is very close to one previous study [4] and higher than another study [1]. Some laboratories, including ours, have implemented robotic specimen arch$\mathrm{ival} /$ retrieval units. As we have shown, the estimated impact of this type of unit on manual time can be substantial, with an estimated reduction of $24.1 \mathrm{hrs} /$ day of manual add-on processing time in handling add-on order requests. However, the timing of add-on orders suggests that improvements on this design may include a combination of short-term/rapidly accessible and longer-term/less accessible specimen storage. Storage of specimens in a rapidly accessible buffer (e.g., very close to the chemistry analyzers) for a limited period of time (e.g., between 8 and 24 hours) would capture the majority of add-on orders. After this time period, specimens can be archived for longerterm storage in a space more distant from the instruments. At that point, turnaround time is likely less important.

The main limitations of our study are that the analysis is retrospective and confined to an academic medical center. The results may not generalize to other hospital or clinic settings. Nevertheless, it is hoped that the results described here provide useful to other institutions attempting to manage the challenges of add-on testing.

\section{Conclusions}

Add-on orders significantly impact the workload of the clinical laboratory. In this study at an academic medical center, the majority of add-on orders were clinical chemistry tests, and most add-on orders occur within 24 hours of original specimen collection. Robotic specimen archival/retrieval units can reduce manual effort in the clinical laboratory associated with add-on orders.

\section{Abbreviations}

A1C: Hemoglobin A1C; BILD: Direct bilirubin; BMP: Basic metabolic panel; CBC: Complete blood count; CPOE: Computerized provider order entry; CRP: C-reactive protein; DIFF: White blood count differential; ED: Emergency department; EHR: Electronic health record; ESR: Erythrocyte sedimentation rate; HAPT: Haptoglobin; HBSG: Hepatitis B surface antigen; HEPC: Hepatitis C antibody; ICU: Intensive care unit; LDH: Lactate dehydrogenase; LFP: Liver function panel; LIS: Laboratory information system; OSMO: Serum/plasma osmolality; PO4MG: Phosphorus and magnesium; PREALB: Prealbumin; PT/ INR: Prothrombin time/international normalized ratio; PTT: Partial thromboplastin time; TAP: Toxic alcohol panel; UIHC: University of lowa Hospitals and Clinics; URIC: Uric acid.

\section{Competing interests}

The authors declare that they have no competing interests.

\section{Authors' contributions}

LSN and MDK were involved in the study concept and design, analysis and interpretation of the data, drafting and revisions of the manuscript. SRD, $\mathrm{RMH}$, and JK assisted with data analysis and interpretation. DRA helped with extraction and analysis of data from the laboratory information system. All authors have read and approved the final manuscript.

\section{Acknowledgements}

MDK thanks the Department of Pathology (Dr. Nitin Karandikar, Department Executive Officer) for providing research funding.

\section{Author details}

'Department of Pathology, University of lowa Hospitals and Clinics, lowa City, IA 52242, USA. ${ }^{2}$ Hospital Computing Information Services, University of lowa Hospitals and Clinics, lowa City, IA 52242, USA. 
Received: 19 March 2015 Accepted: 27 May 2015

Published online: 07 June 2015

\section{References}

1. Melanson SF, Hsieh B, Flood JG, Lewandrowski KB. Evaluation of add-on testing in the clinical chemistry laboratory of a large academic medical center: operational considerations. Arch Pathol Lab Med. 2004;128(8):885-9.

2. Melanson S, Flood J, Lewandrowski K. Add-on testing the clinical laboratory: observations from two large academic medical centers. Lab Med. 2006;37(11):675-8.

3. Kim JY, Kamis IK, Singh B, Batra S, Dixon RH, Dighe AS. Implementation of computerized add-on testing for hospitalized patients in a large academic medical center. Clin Chem Lab Med. 2011;49(5):845-50.

4. Loh TP, Saw S, Sethi SK. Clinical value of add-on chemistry in a large tertiary care teaching hospital. Lab Med. 2012;43(3):82-5.

5. Naumova NN, Schappert J, Kaplan LA. Patterns of add-on tests for hospitalized and for private patient populations. Arch Pathol Lab Med. 2007;131(12):1794-9.

6. Krasowski MD, Chudzik D, Dolezal A, Steussy B, Gailey MP, Koch B, et al. Promoting improved utilization of laboratory testing through changes in an electronic medical record: experience at an academic medical center. BMC Med Inform Decis Mak. 2015;15:11.

7. Krasowski MD, Davis SR, Drees D, Morris C, Kulhavy J, Crone C, et al. Autoverification in a core clinical chemistry laboratory at an academic medical center. J Pathol Inform. 2014;5:13.

\section{Submit your next manuscript to BioMed Central and take full advantage of:}

- Convenient online submission

- Thorough peer review

- No space constraints or color figure charges

- Immediate publication on acceptance

- Inclusion in PubMed, CAS, Scopus and Google Scholar

- Research which is freely available for redistribution 